\title{
Simulating masonry failure using continuous and discontinuous damage models
}

\author{
B. Vandoren \\ Construction Engineering Research Group, Faculty of Engineering Technology, Hasselt University, \\ Hasselt, Belgium
}

\begin{abstract}
In this contribution we propose and compare two finite element models for modelling damage in masonry structures. The first model belongs to the class of discontinuous models, in which masonry failure is modelled through the insertion of discrete displacement jumps at the mortar joints. Discontinuities are incorporated using the Generalised Finite Element Method (GFEM), allowing for an on-the-fly introduction of cracks. The second model is a novel continuous model, in which masonry damage is smeared out over a finite volume. Mesh-sensitivity of strain localisation in this model is regularised by means of a gradient enhanced damage model which takes into account the anisotropy of masonry.
\end{abstract}

\section{INTRODUCTION}

While masonry is known for its simple method of construction, its heterogeneous and quasi-brittle character leads to some interesting challenges for its numerical analysis. Traditionally, numerical masonry models can be categorised into continuous and discontinuous models (Lourenço 1996). In continuous models, masonry damage is smeared out over a certain region, improving the stability of the model but making it difficult to assess the exact crack patterns (Zucchini \& Lourenço 2009). On the other hand, discontinuous models allow for an accurate representation of discrete cracks inside a masonry structure, at the expense of an increased model size. Moreover, discontinuous models are prone to robustness issues, since (parts of the) bricks can become loose from the structure if their surrounding joints are fully damaged, leading to numerical instabilities, i.e. a singular stiffness matrix.

In this contribution we propose an compare two modelling approaches in both categories: (i) a discontinuous model based on the generalised finite element method (Vandoren et al. 2013a) and (ii) a novel continuous finite element model based on the implicit gradient enhanced damage model. Both approaches require additional degrees of freedom which describe the jump fields of the discrete cracks and a regularising non-local strain field, respectively. The performance of both approaches is compared by means of a numerical example of a shear wall test.

\section{DISCONTINUOUS MODELLING OF MASONRY FAILURE USING A GFEM- BASED MODEL}

\subsection{Kinematic framework}

A first approach for modelling masonry failure is using a discrete model in which joints and bricks are modelled as separate entities. These discrete models are often referred to as (simplified) microscale (Lourenço 1996) or mesoscale models (Vandoren et al. 2013b, De Proft et al. 2010). Failure is assumed to localise in the weaker mortar joints, which are represented as zero-thickness entities. These joint elements, which can be considered as 'strong discontinuities' (i.e. a jump in the displacement field), are usually modelled as interface elements located at the edges of the brick elements.

Alternatively, these joint elements can be incorporated in the model as so-called interfacelike' elements using a generalised finite element (GFEM) approach (Simone 2004). The use of a GFEM model offers interesting opportunities for modelling masonry structures with irregular bond patterns, since mortar joints and potential brick cracks do no longer have to coincide with the finite element edges (Simone 2006). Moreover, a GFEM-based masonry model allows mortar joints and other discontinuities to be introduced on-thefly, i.e. only when a critical stress state is exceeded, reducing the total number of degrees of freedom and avoiding the use of high dummy stiffness inside the bricks (Vandoren et al. 2013b). 
When considering the kinematics of a body crossed by a strong discontinuity (Fig. 1), the displacement field can be expressed as

$$
\mathbf{u}=\hat{\mathbf{u}}+H_{\Gamma_{\mathrm{d}}} \tilde{\mathbf{u}}
$$

in which $H_{\Gamma_{\mathrm{d}}}$ is the Heaviside step function and $\hat{\mathbf{u}}$ and $\tilde{\mathbf{u}}$ are the regular and enhanced displacement (or jump) fields, respectively. The value of the Heaviside step function $H_{\Gamma_{d}}$ depends on the location of the considered (integration) point with respect to the discontinuity. It equals unity in the positive domain $\Omega^{+}$and zero on the other side of the discontinuity. The displacement field $\mathbf{u}$ follows from the solution of the discretised generalised finite element equation

$$
\mathbf{K u}=\mathbf{f}
$$

where the stiffness matrix $\mathbf{K}$ is composed as

$$
\mathbf{K}=\left[\begin{array}{ll}
\mathbf{K}_{11} & \mathbf{K}_{12} \\
\mathbf{K}_{21} & \mathbf{K}_{22}
\end{array}\right]
$$

and the displacements and forces are given by

$$
\mathbf{u}=\left\{\begin{array}{l}
\hat{\mathbf{u}} \\
\tilde{\mathbf{u}}
\end{array}\right\}
$$

and

$$
\mathbf{f}=\left\{\begin{array}{l}
\hat{\mathbf{f}} \\
\tilde{\mathbf{f}}
\end{array}\right\},
$$

respectively. In Equation 3, the submatrices read

$$
\begin{aligned}
& \mathbf{K}_{11}=\int_{\Omega} \mathbf{B}^{\mathrm{T}} \mathbf{C}_{\mathrm{b}} \mathbf{B} \mathrm{d} \Omega, \\
& \mathbf{K}_{12}=\mathbf{K}_{21}=\int_{\Omega^{+}} \mathbf{B}^{\mathrm{T}} \mathbf{C}_{\mathrm{b}} \mathbf{B} \mathrm{d} \Omega^{+},
\end{aligned}
$$

and

$$
\mathbf{K}_{22}=\int_{\Gamma_{\mathrm{d}}} \mathbf{N}^{\mathrm{T}} \mathbf{T}_{\mathrm{m}} \mathbf{N} \mathrm{d} \Gamma_{\mathrm{d}}+\int_{\Omega^{+}} \mathbf{B}^{\mathrm{T}} \mathbf{C}_{\mathrm{b}} \mathbf{B} \mathrm{d} \Omega^{+} .
$$

In Equations $6-8, \mathbf{B}$ is the strain-displacement matrix, $\mathbf{N}$ is the matrix containing the finite element shape functions, $\mathbf{T}_{\mathrm{m}}$ is the elastic constitutive matrix of the mortar joints, and $\mathbf{C}_{\mathrm{b}}$ is the elastic constitutive matrix of the bricks.

The equations above are valid for one discontinuity. In the case of a discrete masonry model in which we assume that cracks (i.e. discontinuities)

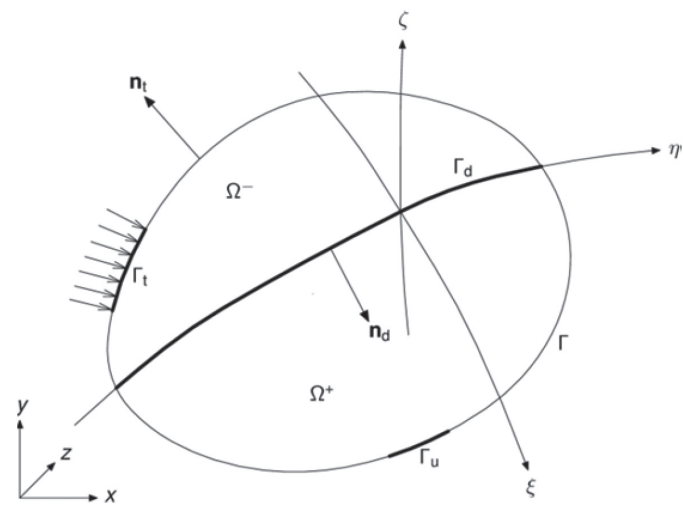

Figure 1. Kinematics of a body $\Omega$ crossed by a strong discontinuity $\Gamma_{\mathrm{d}}$.

only run through the mortar joints, these equations should be modified in order to take into account the kinematics of multiple intersecting discontinuities. In this contribution, we adapt the GFEM model for polycrystals (Simone 2006) in which the displacement field is decomposed according to

$$
\mathbf{u}=\mathbb{\Downarrow}+\sum_{i=1}^{n_{\mathrm{B}}} H_{i} \tilde{\mathbf{u}}_{i}
$$

in which $\tilde{\mathbf{u}}_{i}$ are the enhanced displacements corresponding to brick $i, n_{\mathrm{B}}$ denotes the number of bricks in the masonry structure, and $H_{i}$ is the Heaviside step function belonging to brick $i$ which equals one inside the brick and zero in the rest of the domain. In other words, the displacement field of each brick are governed by a designated set of degrees of freedom $\tilde{\mathbf{u}}_{i}$. It should be noted that joints can be deactivated (which means that bricks are numerically 'glued' together) by removing the corresponding enhanced degrees of freedom from the system of equations (Eq. 2). This can be of interest when trying to avoid rigid body modes of 'loose' bricks and thus improving the conditioning of the system matrix $\mathbf{K}$.

\subsection{Constitutive model}

In the proposed discontinuous model, bricks remain linear elastic whereas the nonlinear joint behaviour is governed by a simple isotropic damage law:

$\mathbf{t}=(1-\omega) \mathbf{T}_{\mathrm{m}}\|\mathbf{u}\|$

in which $\mathbf{t}$ and $\|\mathbf{u}\|$ are, respectively, the tractions and displacement jumps at the joint (consisting of a normal and tangential component) and $\omega$ is a 


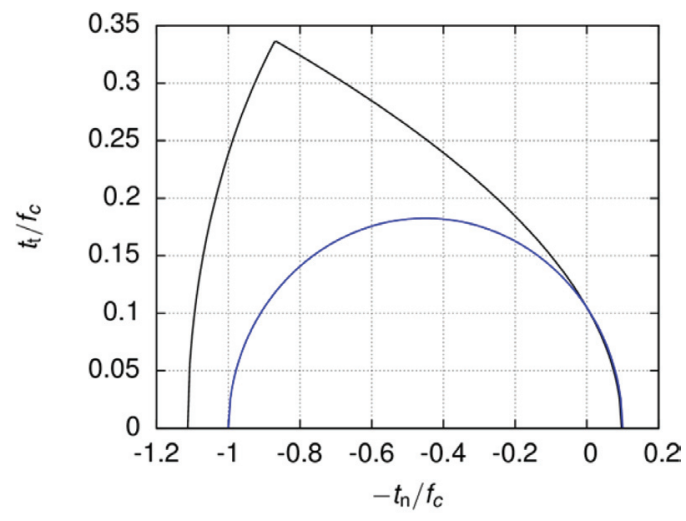

degenerated Drucker-Prager, $\nu_{\mathrm{m}}=0.15$ degenerated Drucker-Prager, $\nu_{\mathrm{m}}=0.0$

Figure 2. Degenerated Drucker-Prager damage criterion in normalised traction space $\left(f_{\mathrm{c}}\right.$ denotes the compressive strength, $v_{\mathrm{m}}$ is the Poisson's ratio of the mortar joint, $t_{\mathrm{t}}$ and $t_{\mathrm{n}}$ are the tangential and normal traction, respectively).

damage parameter varying between zero (no damage) and unity (fully damaged state), which is calculated according to

$$
\omega=1-\frac{\kappa_{0}}{\kappa} \exp \left(-\frac{\kappa-\kappa_{0}}{\gamma}\right)
$$

in which $\gamma$ governs the brittleness (which is calculated using the fracture energy $G_{\mathrm{f}}$, see (Feenstra 2002)), $\kappa_{0}$ is the damage threshold, i.e. the value of the equivalent jump $\|u\|_{\text {eq }}$ at which damage initiates. $\kappa$ is a history parameter which memorises the maximum value ever attained of the equivalent jump. The latter is governed by a material model which is a degenerated Drucker-Prager criterion, see Figure 2 and (Vandoren et al. 2013b).

\section{CONTINUOUS MODELLING OF MASONRY FAILURE USING A GRADIENT-ENHANCED DAMAGE MODEL}

\subsection{Kinematic framework}

When modelling larger masonry structures, it may no longer be feasible to use a discontinuous masonry model with a discrete representation of bricks and mortar joints. Instead, failure of the orthotropic masonry composite can be modelled in a continuous manner, without the need of inserting discontinuities within the finite element mesh. Since local damage models (e.g. Eq. 11) will lead to mesh-dependent results (i.e. damage will localise to the smallest numerical entity), a so-called localisation limiter should be employed. This localisation limiter introduces an objective length scale into the model, which governs the volume of localisation. In most continuous masonry models, meshdependency of strain softening is regularised using Cosserat continua (Addessi et al. 2010) or non-local models (Marfia \& Sacco 2012). In this contribution, we use an implicit gradient-enhanced damage model for the objective description of strain localisation and failure (Peerlings et al. 1996). To the best of the author's knowledge, this approach has never been adopted for the macroscopic modelling of masonry failure. Moreover, the model has been improved by taking into account an anisotropic strain averaging volume, whereas traditional gradient-enhanced damage models employ an isotropic interaction kernel. In this model, the discretised system of finite element equations reads:

$\mathbf{K u}=\mathbf{f}$

in which the stiffness matrix $\mathbf{K}$ equals

$\mathbf{K}=\left[\begin{array}{ll}\mathbf{K}_{11} & \mathbf{K}_{12} \\ \mathbf{K}_{21} & \mathbf{K}_{22}\end{array}\right]$

and the vector containing the unknown degrees of freedom is given by

$\mathbf{u}=\left\{\begin{array}{c}\hat{\mathbf{u}} \\ \bar{\varepsilon}_{\mathrm{eq}}\end{array}\right\}$,

in which $\bar{\varepsilon}_{\mathrm{eq}}$ is the (nodal) non-local equivalent strain field, which can be considered as a variable which smoothens and averages the local equivalent strain field $\boldsymbol{\varepsilon}_{\mathrm{eq}}$ calculated in each integration point in each element. The right-hand side of Equation 12 equals

$\mathbf{f}=\left\{\begin{array}{l}\mathbf{f} \\ \tilde{\mathbf{f}}\end{array}\right\}$

in which

$\Phi=\int_{\Omega} \mathbf{N}^{\mathrm{T}} \bar{\varepsilon}_{\text {eq }} \mathrm{d} \Omega$.

The components of the stiffness matrix are given by

$\mathbf{K}_{11}=\int_{\Omega} \mathbf{B}^{\mathrm{T}} \mathbf{C}_{\mathrm{h}} \mathbf{B} \mathrm{d} \Omega$, 
$\mathbf{K}_{12}=\mathbf{K}_{21}=\mathbf{0}$,

and

$$
\mathbf{K}_{22}=\int_{\Omega} \mathbf{N}^{\mathrm{T}} \mathbf{N}+\mathbf{B}^{\mathrm{T}} \mathbf{c B} \mathrm{d} \Omega .
$$

Equation 12 thus represents two equations which are both dependent on $\boldsymbol{\varepsilon}_{\text {eq }}$. Linearising these equations, which is necessary for a Newton-Raphson solution procedure, results in a numerical coupling of the equations, see (Peerlings et al. 1996) for more details. $\mathbf{C}_{\mathrm{h}}$ in Equation 17 represents the homogenised elastic constitutive matrix of the masonry material, as will be explained in Section 3.2.1. In Equation 19, $\mathbf{c}$ is a matrix containing length-scale parameters, see Section 3.2.2.

\subsection{Constitutive model}

\subsubsection{Elastic regime: homogenisation of elastic material properties}

Since mortar joints and brick are no longer represented by separate entities in the model, the elastic properties of both constituents should be homogenised. In the proposed masonry model, the homogenised stiffness is calculated according to the periodic eigenstrain homogenisation technique by Wang et al. (2007):

$$
\mathbf{C}_{\mathrm{h}}=\mathbf{C}_{\mathrm{m}}\left[\mathbf{I}-F_{b}\left(\left(\mathbf{C}_{\mathrm{m}}-\mathbf{C}_{\mathrm{b}}\right)^{-1} \mathbf{C}_{\mathrm{m}}-\mathbf{S}_{\mathrm{m}}\right)^{-1}\right]
$$

in which $\mathbf{C}_{\mathrm{m}}$ is the constitutive matrix of the mortar joints, $\mathbf{I}$ is the identity matrix, $F_{\mathrm{b}}$ is the volume fraction of the bricks, and $\mathbf{S}_{\mathrm{m}}$ is an Eshelby tensor which is dependent on the masonry bond and dimensions of the masonry constituents. The reader is referred to the article of Wang et al. (2007) for a comparison with other elastic homogenisation methods.

\subsubsection{Inelastic regime: gradient-enhanced damage model}

As outlined in Section 3.1, the implicit gradientenhanced damage model by Peerlings et al. (1996) will be adapted in this study. Analogous to Equation 10, the stress-strain relation is given by an isotropic damage law:

$$
\sigma=(1-\omega) \mathbf{C}_{\mathrm{h}} \varepsilon
$$

in which the damage parameter $\omega$ is evaluated using Equation 11. However, the history parameter $\kappa$ and thus material damage is no longer governed by a local strain measure such as $\|u\|_{\text {eq }}$ or $\varepsilon_{\text {eq }}$.
Instead, a non-local equivalent strain $\bar{\varepsilon}_{\text {eq }}$ is used, which is calculated according to

$$
\bar{\varepsilon}_{\text {eq }}-c \nabla^{2} \bar{\varepsilon}_{\text {eq }}=\varepsilon_{\text {eq }}
$$

in which $c$ is a length scale parameter which defines an isotropic interaction kernel or averaging domain, see (Peerlings et al. 1996) for more details. Alternatively, as proposed by Simone (2013), an anisotropic interaction kernel can be obtained by modifying Equation 22 into:

$\overline{\mathcal{E}}_{\mathrm{eq}}-\nabla\left(\mathbf{c} \nabla \bar{\varepsilon}_{\mathrm{eq}}\right)=\varepsilon_{\text {eq }}$

in which, as mentioned in Section 3.1, $\mathbf{c}$ is a matrix containing length-scale parameters. In our model, this matrix is not only dependent on the stress state, but also on the morphology of the structure, i.e. the masonry bond. It is calculated according to

$\mathbf{c}=\left[\begin{array}{cc}c_{x x} & 0 \\ 0 & c_{y y}\end{array}\right]$,

in which

$$
\begin{aligned}
& c_{x x}=-g\left(\frac{c_{\max }-c_{\min }}{2}\right)+\left(\frac{c_{\max }+c_{\min }}{2}\right), \\
& c_{y y}=g\left(\frac{c_{\max }-c_{\min }}{2}\right)+\left(\frac{c_{\max }+c_{\min }}{2}\right),
\end{aligned}
$$

and

$$
c_{\max }=n c_{\min }=c\left|\frac{\sigma_{\mathrm{eq}}}{f_{\mathrm{t}}}\right|,
$$

where $f_{\mathrm{t}}$ is the tensile strength and $\sigma_{\mathrm{eq}}$ is the equivalent stress, which is, like the local equivalent strain $\varepsilon_{\text {eq }}$ in Equation 23, governed by a continuum Drucker-Prager criterion (Massart et al. 2004), see Figure 3. In Equations 25-26, $g$ is a parameter which takes into account the geometry of the masonry structure: it equals 1 for head joints, -1 for bed joints and 0 inside the bricks. This parameter orients the interaction kernel in the direction of the dominant microcrack (Fig. 4) and forms a so-called 'groove field', as depicted in Figure 5. This field reflects the position and thickness of the mortar joints and defines the preferential failure paths in the masonry wall. In other words, this field incorporates orthotropy in the inelastic range by taking into account the masonry bond when calculating the damage-driving parameter $\left(\bar{\varepsilon}_{\text {eq }}\right)$ in Equation 23. Parameter $g$ does not only play a role in damage propagation, but can also be used 


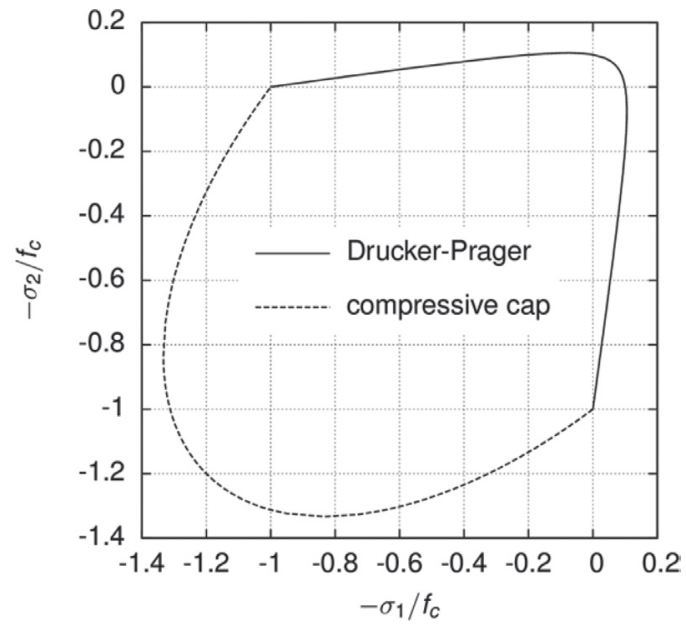

Figure 3. Continuum Drucker-Prager damage criterion in normalised stress space ( $f_{c}$ denotes the compressive strength, $\sigma_{1}$ and $\sigma_{2}$ are the principal stresses).

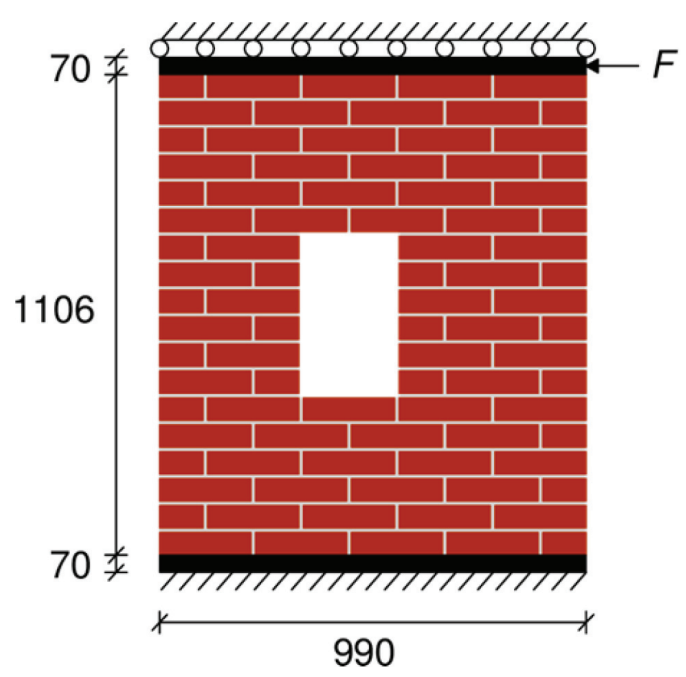

Figure 4. Shear wall test: setup. All dimensions in mm.

to predefine the location of damage initiation by locally reducing the damage threshold and stiffness according to

$\kappa_{0}{ }^{\prime}=(1-\alpha|g|) \kappa_{0}$

and

$$
\mathbf{C}_{\mathrm{h}}{ }^{\prime}=(1-\beta|g|) \mathbf{C}_{\mathrm{h}},
$$

respectively.
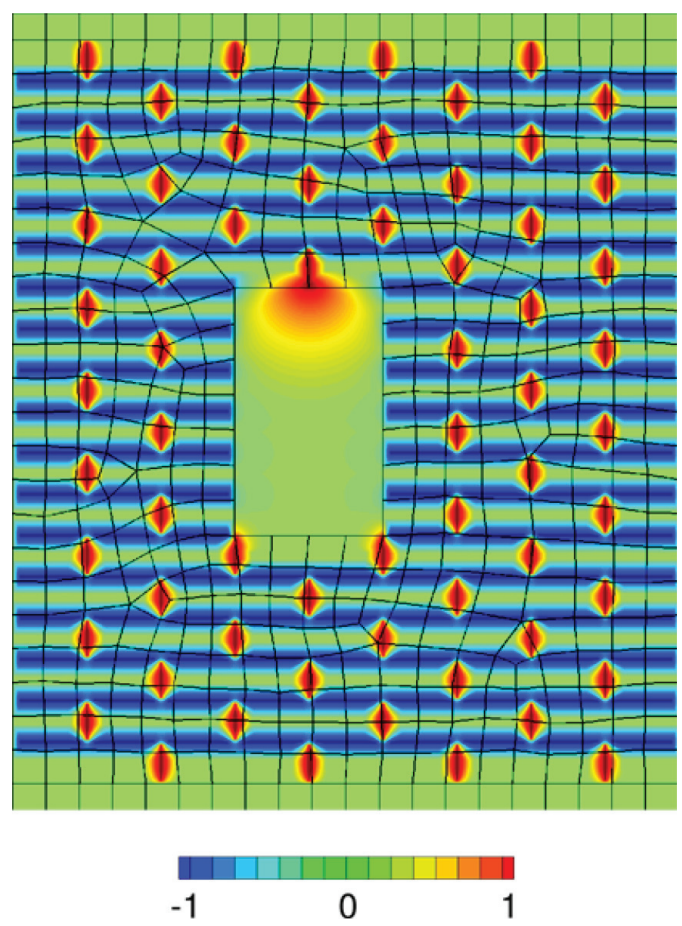

Figure 5. Shear wall test: groove field $g$ and mesh. This groove field, which reflects the position and thickness of the mortar joints, defines the preferential failure paths in the masonry wall, as explained in Section 3.2.2.

Table 1. Material parameters for the shear wall test.

\begin{tabular}{lll}
\hline Parameter & Bricks & Joints \\
\hline Dimensions & $52\left(210\left(100 \mathrm{~mm}^{3}\right.\right.$ & $10 \mathrm{~mm}$ \\
E & $11,000 \mathrm{~N} / \mathrm{mm}^{2}$ & $2200 \mathrm{~N} / \mathrm{mm}^{2}$ \\
$v$ & 0.20 & 0.25 \\
$f_{\mathrm{t}}$ & $(*)$ & $0.25 \mathrm{~N} / \mathrm{mm}^{2}$ \\
$f_{\mathrm{c}}$ & $\left(^{*}\right)$ & $2.5 \mathrm{~N} / \mathrm{mm}^{2}$ \\
$G_{\mathrm{f}}$ & $(*)$ & $0.018 \mathrm{Nmm} / \mathrm{mm}^{2}$ \\
\hline
\end{tabular}

(*) Bricks remain linear elastic in the discontinuous model. In the continuous model, the inelastic parameters of the joints are used.

\section{NUMERICAL EXAMPLE: SHEAR WALL}

The performance of both models is assessed through the numerical analysis of a shear wall, which material parameters and test setup are given by Table 1 and Figure 4, respectively. Groove field $g$ of the continuous model is depicted in Figure 5. Although of completely different nature, both models are able to capture the typical failure pattern observed in experiments (Raijmakers \& Vermeltfoort 1992), see Figures 6-12. However, it should be noted 


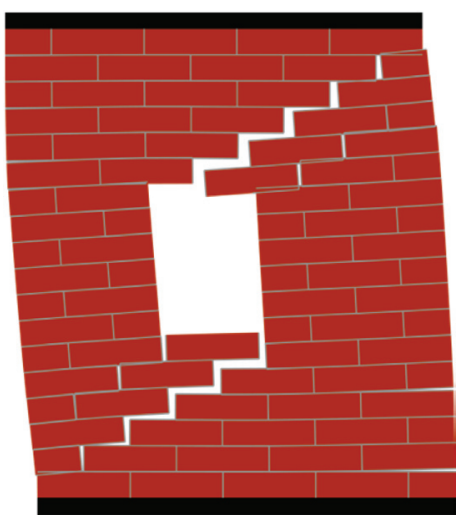

Figure 6. Shear wall test-discontinuous model: crack paths and deformations for $u=0.50 \mathrm{~mm}$.

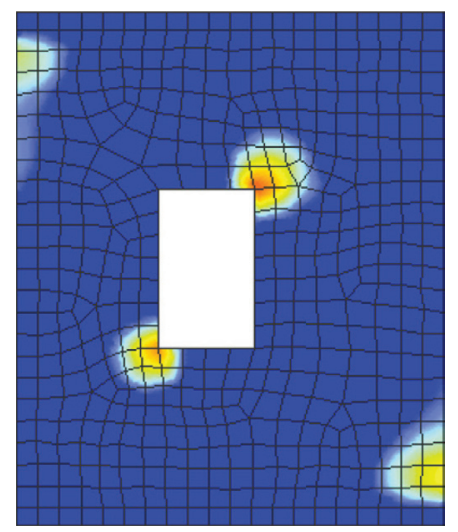

Figure 7. Shear wall test-continuous model: damage fields for $u=0.10 \mathrm{~mm}$ and $\alpha=0$.

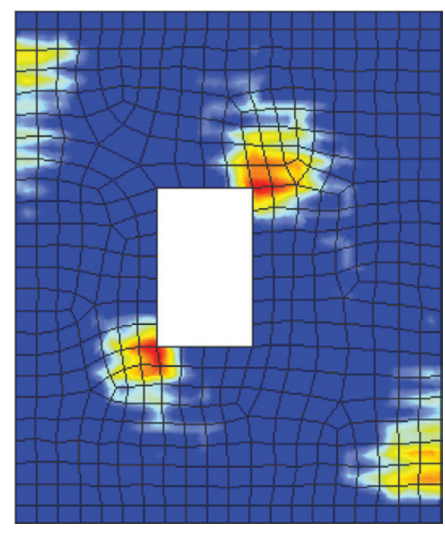

Figure 8. Shear wall test - continuous model: damage fields for $u=0.10 \mathrm{~mm}$ and $\alpha=0.5$.

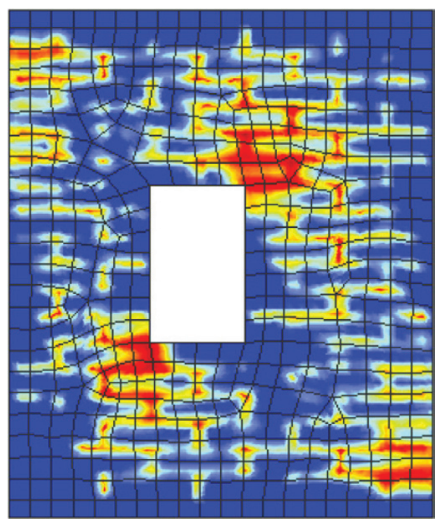

Figure 9. Shear wall test-continuous model: damage fields for $u=0.10 \mathrm{~mm}$ and $\alpha=0.99$.

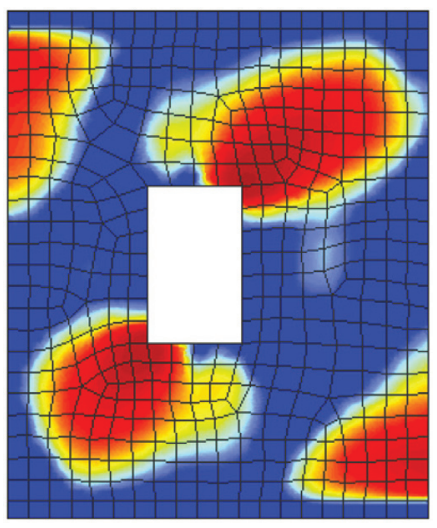

Figure 10. Shear wall test-continuous model: damage fields for $u=0.50 \mathrm{~mm}$ and $\alpha=0$.

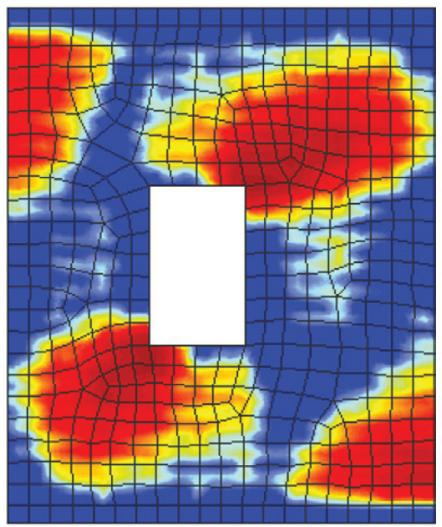

Figure 11. Shear wall test-continuous model: damage fields for $u=0.50 \mathrm{~mm}$ and $\alpha=0.5$. 
that, in contrast with the experiments, no vertical precompression has been applied prior to the shear loading. Due to its discontinuous character, the GFEM-based model exhibits a tortuous load-displacement behaviour whereas all load-displacement curves of the continuous model remain smooth, see Figure 13. Moreover, the discontinuous models fails after an imposed displacement of $0.6 \mathrm{~mm}$, while no convergence problems were encountered with the continuous model. In this model, the parameter $\alpha$

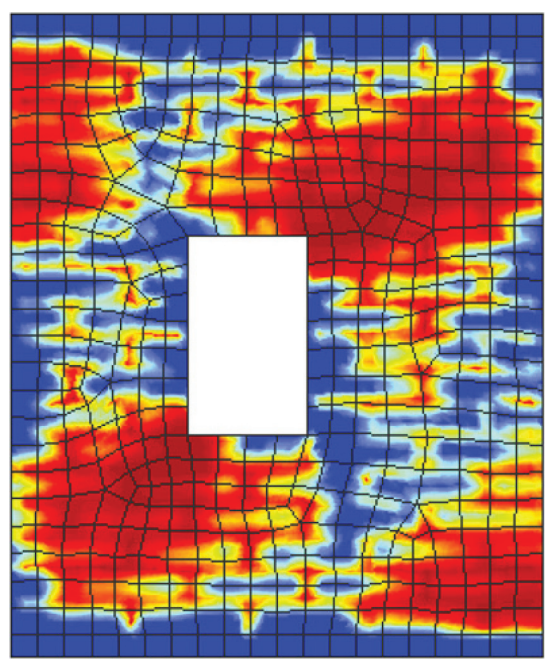

Figure 12. Shear wall test-continuous model: damage fields for $u=0.50 \mathrm{~mm}$ and $\alpha=0.99$. can be regarded as a 'focussing' parameter: a low value results in a smeared damaging zone, whereas a higher value leads to a sharpened image with distinct failure patterns. However, a high value reduces the overall strength of the masonry wall, as can be observed in Figure 13. In order to maintain the peak load of the structure, the groove field $g$ can be shifted, i.e. the modified damage threshold $\kappa_{0}$ ' in Equation 28 is locally set higher for points inside the bricks, leading to a higher average strength of the system (denoted with 'shifted groove field' in Fig. 13). It should be noted that the current continuous model leads to an excessive damage spread when compared to results of other authors, indicating that the proposed model requires further calibration of the length scale parameters $c_{\max }$ and $c_{\min }$ in Equation 27.

\section{CONCLUSION AND FUTURE WORKS}

In this contribution, we presented two approaches for modelling damage in masonry structures: a discontinuous model based on the generalised finite element method and a continuous model based on the implicit gradient enhanced damage model. We demonstrated the performance of both models through an academic test case. Future work will focus on the development of a hybrid discontinuous-continuous model which combines the advantages of the presented models, i.e. numerical robustness (continuous model) and accurate representation of the crack paths (discontinuous model).

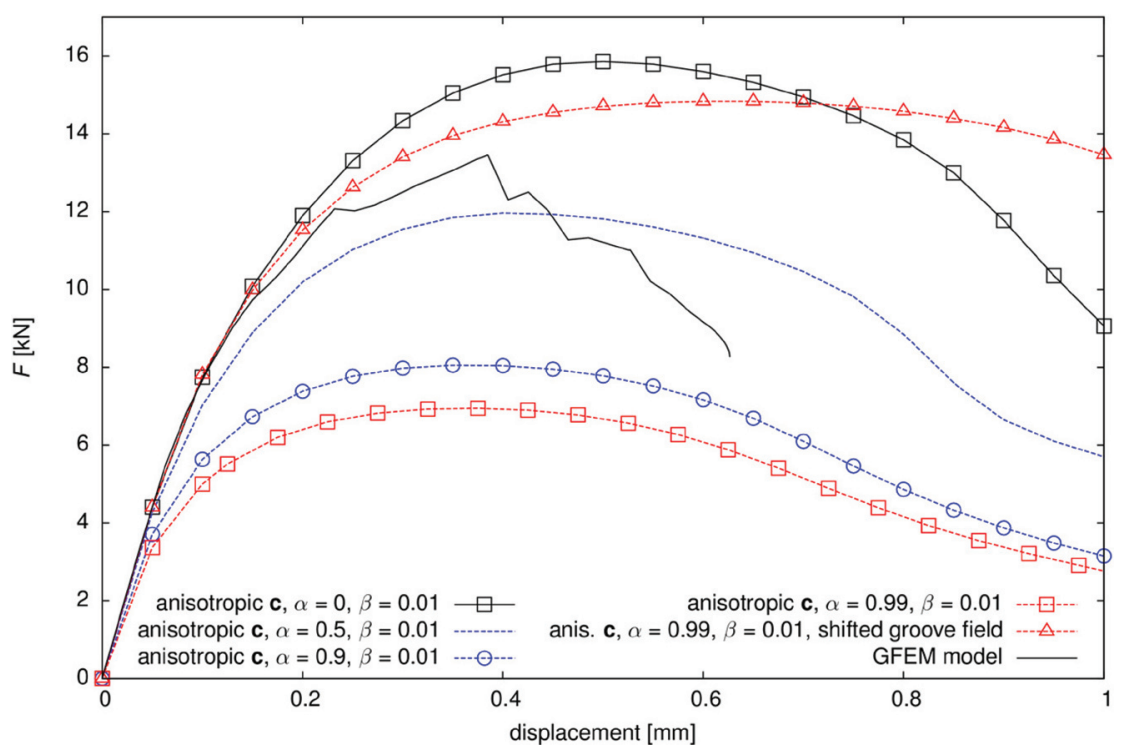

Figure 13. Shear wall test: force-displacement diagrams for the discontinuous (GFEM) and continuous masonry models. 
We will also incorporate more realistic constitutive laws, which take into account cyclic loading and dilatancy behaviour.

\section{REFERENCES}

Addessi, D., Sacco, E., \& Paolone, A. 2010. Cosserat model for periodic masonry deduced by nonlinear homogenization. Eur. J. Mech. A/Solids 29:724-737.

De Proft, K., Heyens, K., \& Sluys, L.J. 2010. Mesoscopic modelling of masonry failure. Proceedings of the ICEEngineering and Computational Mechanics 164:41-46.

Feenstra, P.H. 2002. Implementing an isotropic damage model in Diana. Use-case for the user-supplied subroutine usrmat, In Proceedings of the Third DIANA World Conference.

Lourenço, P.B. 1996. Computational strategies for masonry structures, $\mathrm{PhD}$ thesis, Delft University of Technology.

Marfia, S. \& Sacco, E. 2012. Multiscale damage contactfriction model for periodic masonry walls. Comput. Methods Appl. Mech. Engrg. 205-208:189-203.

Massart, T.J., Peerlings, R.H.J., \& Geers, M.G.D. 2004. Mesoscopic modeling of failure and damage-induced anisotropy in brick masonry, Eur. J. Mech. A/Solids 23:719-735.

Peerlings, R.H.J., de Borst, R., Brekelmans, W.A.M., \& de Vree J.H.P. 1996. Gradient-enhanced damage for quasi-brittle materials. Int. J. Numer. Meth. Engrg. 39:3391-3403.
Raijmakers, T.M.J. \& Vermeltfoort, A.T. 1992. Deformation controlled tests in masonry shear walls-Report B-92-1156, Technical report, TNO Bouw.

Simone, A. 2004. Partition of unity-based discontinuous elements for interface phenomena: computational issues. Commun. Numer. Meth. Engng. 20:465-478.

Simone, A., Duarte, C.A., \& Van der Giessen, E 2006. A generalized finite element method for polycrystals with discontinuous grain boundaries. Int. J. Numer. Methods Engrg. 67:1122-1145.

Simone, A. 2013. Advances in gradient-enhanced damage models with evolving length scales. In Proceedings of The Third International Conference on Computational Modeling of Fracture and Failure of Materials and Structures.

Vandoren, B., De Proft, K., Simone, A., \& Sluys, L.J. 2013b. Modelling crack initiation and propagation in masonry using the partition of unity method. In Proceedings of the VIII International Conference on Fracture Mechanics of Concrete and Concrete Structures.

Vandoren, B., De Proft, K., Simone, A., \& Sluys, L.J. 2013a. Mesoscopic modelling of masonry using weak and strong discontinuities. Comput. Methods Appl. Mech. Engrg. 255:167-182.

Wang, G., Li, S., Nguyen, H.-N., \& Sitar, N. 2007. Effective Elastic Stiffness for Periodic Masonry Structures via Eigenstrain Homogenization. Journal of Materials in Civil Engineering vol. March 2007:269-277.

Zucchini, A., \& Lourenço, P.B. 2009. A micro-mechanical homogenisation model for masonry: Application to shear walls. Int. J. Solids Struct. 46:871-886. 